\title{
GA-A24523
}

\section{MODELING OF FEEDBACK AND ROTATION STABILIZATION OF THE RESISTIVE WALL MODE IN TOKAMAKS}

\author{
by \\ M.S. CHU, A. BONDESON, M.S. CHANCE, Y.Q. LIU, \\ A.M. GAROFALO, A.H. GLASSER, G.L. JACKSON, R.J. LA HAYE, \\ L.L. LAO, G.A. NAVRATIL, M. OKABAYASHI, H. REIMERDES, \\ J.T. SCOVILLE, and E.J. STRAIT
}




\section{DISCLAIMER}

This report was prepared as an account of work sponsored by an agency of the United States Government. Neither the United States Government nor any agency thereof, nor any of their employees, makes any warranty, express or implied, or assumes any legal liability or responsibility for the accuracy, completeness, or usefulness of any information, apparatus, product, or process disclosed, or represents that its use would not infringe privately owned rights. Reference herein to any specific commercial product, process, or service by trade name, trademark, manufacturer, or otherwise, does not necessarily constitute or imply its endorsement, recommendation, or favoring by the United States Government or any agency thereof. The views and opinions of authors expressed herein do not necessarily state or reflect those of the United States Government or any agency thereof. 


\title{
MODELING OF FEEDBACK AND ROTATION STABILIZATION OF THE RESISTIVE WALL MODE IN TOKAMAKS
}

\author{
by \\ M.S. CHU, A. BONDESON, ${ }^{*}$ M.S. CHANCE, ${ }^{\dagger}$ Y.Q. LIU, ${ }^{*}$ \\ A.M. GAROFALO, ${ }^{\ddagger}$ A.H. GLASSER, ${ }^{\perp}$ G.L. JACKSON, R.J. LA HAYE, \\ L.L. LAO, G.A. NAVRATIL, ${ }^{\ddagger}$ M. OKABAYASHI, ${ }^{\dagger}$ H. REIMERDES, ${ }^{\ddagger}$ \\ J.T. SCOVILLE, and E.J. STRAIT
}

This is a preprint of a paper to be submitted for publication in Phys. Plasmas.

*Chalmers University of Technology, Göteborg, Sweden.

${ }^{\dagger}$ Princeton Plasma Physics Laboratory, Princeton, New Jersey.

${ }^{\ddagger}$ Columbia University, New York, New York.

${ }^{\Delta}$ Los Alamos National Laboratory, Los Alamos, New Mexico.

Work supported by

the U.S. Department of Energy under

DE-FG03-95ER54309, DE-AC02-76CH03073,

and DE-FG02-89ER54461 



\begin{abstract}
Steady-state operation of the advanced tokamak reactor relies on maintaining plasma stability with respect to the resistive wall mode (RWM). Active magnetic feedback and plasma rotation are the two methods proposed and demonstrated for this purpose. A comprehensive modeling effort including both magnetic feedback and plasma rotation is needed for understanding the physical mechanisms of the stabilization and to project to future devices. For plasma with low rotation, a complete solution for the feedback issue is obtained by assuming the plasma obey ideal magnetohydrodynamics (MHDs) and utilizing a normal mode approach (NMA) [M.S. Chu, et al., Nucl. Fusion 43, 441 (2003)]. It is found that poloidal sensors are more effective than radial sensors and coils inside of the vacuum vessel more effective than outside. For plasmas with non-negligible rotation, a comprehensive linear non-ideal MHD code, the MARS-F has been found to be suitable. MARS-F [Y.Q. Liu, et al., Phys. Plasmas 7, 3681 (2000)] has been benchmarked in the ideal MHD limit against the NMA. Effect of rotation stabilization of the plasma depends on the plasma dissipation model. Broad qualitative features of the experiment are reproduced. Rotation reduces the feedback gain required for RWM stabilization. Reduction is significant when rotation is near the critical rotation speed needed for stabilization. International Thermonuclear Experimental Reactor (ITER) [R. Aymar, et al., Plasma Phys. Control. Fusion 44, 519 (2002)] (scenario IV for advanced tokamak operation) may be feedback stabilized with $\beta$ above the no wall limit and up to an increment of $\sim 50 \%$ towards the ideal limit. Rotation further improves the stability.
\end{abstract}





\section{INTRODUCTION}

The advanced tokamak ${ }^{1,2}$ provides an attractive reactor design concept which operates in steady state, at high power density and simultaneously maximizes plasma $\beta$, energy confinement time and the fraction of bootstrap current. The $\beta$ limit in advanced tokamaks are invariably set by low toroidal mode number external kink modes which can be stabilized by placing a perfectly conducting wall suffciently close to the edge of the plasma. However, the stabilizing external wall possesses a small but finite resistivity. This converts the external kink mode into a slowly growing magnetohydrodynamic (MHD) mode - the resistive wall mode (RWM), which can destroy the plasma. It has now been demonstrated that both rotation and magnetic feedback can stabilize the $\mathrm{RWM}^{3-5}$. A comprehensive stability model which includes both plasma rotation and magnetic feedback is needed to understand the results from present day experiments and predict the performance of feedback stabilization in future devices such as the International Thermonuclear Experimental Reactor (ITER). ${ }^{6}$

To model the performance of the tokamak under the feedback environment, it is necessary to know the response of the plasma to an arbitrary electromagnetic perturbation in the wavelength and frequency range of interest. Once this response is known, i.e. the physics model is identified, the detailed behavior of the system during feedback is in principle completely determined. Up to now, various efforts have been expended towards the construction of comprehensive tools for studying the feedback stabilization of the RWM. This paper provides an account and comparison of two of these efforts and assess their status of validity and reality.

In the limit when the non-ideal effects such as plasma dissipation and rotation are negligible, the plasma response to external electromagnetic perturbationis is completely determined by the usual ideal MHD equations and is well known. Thus for an ideal MHD plasma, we can have a complete and detailed model for feedback. This feedback model has been formulated. ${ }^{7}$ For tokamak geometry, it is implemented by coupling the $\mathrm{DCON}^{8}$ code, the generalization of the VACUUM ${ }^{9}$ code and a feedback code. This code package, the NMA, ${ }^{7}$ uses the normal mode approach and utilizes the orthonormal eigenfunctions of the open loop system as part of the dynamical feedback phase space. Since the plasma dynamics in the open loop operation is first obtained completely, the details of the feedback, such as the transfer functions, can in principle be obtained completely. There is no conceptual problem in a complete simulation of the feedback process, including the multiple input, multiple output operations. The NMA package has been utilized to study the stability of a generic set of equilibria in the feedback geometry implemented in DIII-D. ${ }^{10}$ It has been found that poloidal sensors are superior to radial sensors and placing the feedback coils inside is more effective than outside of the vacuum vessel. The description of the normal mode approach towards the feedback of an ideal plasma is given in section II.

Present day tokamaks are uaually heated by neutral beams and are rotating at frequencies

that are found to have a substantial effect on the stability of the plasma to the RWM. The effect of feedback on such plasmas cannot be studied by using the normal mode approach. 
A more general method is needed. The rotational stabilization of the plasma comes from the effect of dissipation coupled with the plasma rotation that give rise a phase shift to the mode. This effect was first pointed out by Bondeson and Ward ${ }^{11}$ and has been subsequently demonstrated conclusively in DIII-D experiments. ${ }^{4}$ Physically, this stabilization implies that in a rotating plasma, the dispersion relation of RWM specifies both the growth rate and the freqeuncy at which the energy and momentuem exchanged between the plasma and the resistive wall can be conserved. With no plasma rotation, and a stationary wall, the RWM is not expected to transmit angular momentum between the plasma and the wall - the conservation of angular momentum is guaranteed, only the energy exchange between the plasma and the resistive wall need to be considered. This can always proceed at a particular (growth) rate. However, at large plasma rotations, both the energy and angular momentum exchanges have to be dealt with. Stabilization results from the conservation of both the energy and angular momentum can not be arranged at any complex frequency. This process cannot be described by the usual ideal MHD for a static plasma and is outside of the scope of the NMA. The linear non-ideal MHD stability code MARS, ${ }^{12}$ which solves for the eigenvalue of the complex frequency in a rotating plasma can be utiltized to study the stability of plasma with rotation. However, the complete modeling of this process requires the detailed knowledge of the angular momentum exchange between the plasma and the resistive wall. This depends not only on the resistivity of the resisitve wall, which is known, but also the the dissipation process in the plasma, which is yet unknown. In this work, two dissipation models are employed: the ion sound wave damping model and the kinetic damping model. The predicted topology of the stability phase space has been computed for the set of generic equilbiria and compared with the experiment. Broad agreements have been found for this topology using either damping models, with a slight preference towards the ion sound wave damping. However, due to the difference in the equilbria employed, it is not yet possible to differentiate between the different models. Nevertheless, this comparison gives us a measure of validity of the models. As far as feedback is concerned, it is important to note that the open loop stability does not possess a complete spectrum of eigenvalues with orthonormal eigenfunctions. Only the least stable eigenvalue with its eigenmode structure is known. The rotation stabilization of the plasma is discussed in section III.

The effect of feedback on the stabilization of the RWM involves the use of sensors and feedback coils to modify the stability of the plasma. The open loop stability problem studied by MARS needed to be modified to include these sensors and feedback coils - closing the loop on the plasma feedback process - to the (new) closed loop stability problem. This important step has been accomplished in MARS by implementing the external coil as sources and the placement of sensor coils into the system - the MARS-F code. ${ }^{13}$ Therefore MARS-F solves for the closed loop stability of the plasma together with its feedback configuration. For this, the information about the sensor loops, the feedback coils, and the gain values, all have to be given first. The complete stability of the open loop plasma response is inferred from a (dense) set of closed loop stability results with different feedback gains and the Pade approximation. ${ }^{14}$ To compare the validity of these two different approaches of MARS-F and 
NMA, MARS-F was used to study the feedback stability of the reference set of equilibria, neglecting the non-ideal effects. It is found that MARS-F gives similar feedback predictions for the reference set of equilibria as NMA. MARS-F is then utilized to study the stabilization of a rotating plamsa. It is found that plasma rotation reduces the growth rate of RWM. The effect of rotation induced feedback stabilization is most significant when the flow has reduced the growth rate substantially before the feedback gain has reached the value of 1 or 2. (In here the feedback gain is defined as the ratio of the perturbation field detected at the sensor due to the feedback coil vs. the total field detected at the sensor.) Then feedback can successfully stabilize the mode. Feedback stabilization of a rotating plasma is discussed in section IV.

Lastly, the MARS-F code is applied ${ }^{15}$ to the ITER design. It is shown that the present design can stabilize the resistive wall mode with $\beta$ value above the design value. The effect of flow can provide even more stability margin to the design. The feedback stabilization of ITER is discussed in section V.

A brief discussion and summary is given in section VI. 


\section{NORMAL MODE APPROACH TO FEEDBACK STABILIZATION OF AN \\ IDEAL PLASMA}

Plasmas in future reactors are expected to rotate with negligible rotation speed. Feedback stabilization of plasmas with no or negligible rotation is therefore of particular interest. In this case, the plasma obeys ideal MHD equations. Without feedback, the dynamics is completely determined by a set of normal modes. The behavior of feedback can be completely described in terms of the interaction between the feedback coils with this set of normal modes.

Central to this approach is the consideration of the energy conservation relation between the various components of the system. This is a functional quadratic in the perturbed plasma displacement $\xi$ in the plasma and the perturbed magnetic fields $\delta B$ in the outside 'vacuum' region.

$$
\delta W_{p}+\delta K+\delta W_{v}+D_{w}+\delta E_{c}=0
$$

In Eq. (1), $\delta W_{p}$ is the perturbed plasma potential energy, $\delta K$ is the kinetic energy, $\delta W_{v}$ the perturbed vacuum energy, $D_{w}$, the dissipation energy in the resistive wall and $\delta E_{c}$ the energy exchange between the feedback coil and the plasma resistive wall system. A schematic of this geometry which is appropriate for the DIII-D feedback configuration is shown in Fig. 1. For resistive wall mode, the frequency is very low, the kinetic energy term is negligible. During the open loop operation $\delta E_{c}=0$, Eq. (1) is self-ajoint and determines a set of normal modes, $\left\{\xi_{i}, \delta B_{i}\right\}$, with growth rates $\left\{\gamma_{i}\right\}$ and with $D_{w}$ being the norm. This is an energy principle extended from that of the usual ideal MHD energy principle with $D_{w}$ replacing the plasma kinetic energy. Only one of these normal modes(the RWM), can be unstable. Shown in Fig. 2 is the distribution of the eddy current of the unstable RWM on the surface of the resistive wall unfolded into the toroidal-poloidal plane. It is seen that the perturbed current is concentrated near the poloidal angle $\pi$, i.e. the outboard midplane. The rest of the open loop eigenfunctions are stable (damped) modes in which the resistive wall provides the dissipation. During the closed loop operation, the feedback currents and $\delta E_{c}$ are non zero, the requirement of Eq. (1) then demands that the amplitude of the normal modes $\left\{\alpha_{i}\right\}$ are determined by

$$
\frac{\partial \alpha_{i}}{\partial t}-\gamma_{i} \alpha_{i}=E_{i}^{c} I_{c}
$$

In Eq. (2), $\left\{E_{i}^{c}\right\}$ is the excitation matrix which describes the excitation of the eigenmode $\left(\xi_{i}, \delta B_{i}\right)$ by the feedback current $I_{c}$. The circuit equations for the currents $I_{c}$ incorporates the feedback logic

$$
\frac{\partial I_{c}}{\partial t}+\frac{1}{\tau_{c c^{\prime}}} I_{c^{\prime}}=G_{c l} F_{l i}\left(\alpha_{i}\right)
$$




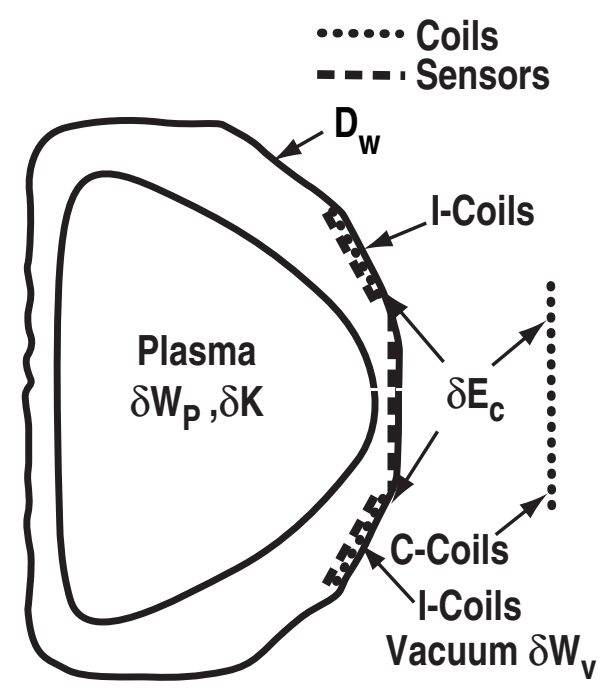

Fig. 1. Schematic of the feedback geometry in DIII-D. The plasma, which contributes to the potential energy $\delta W_{p}$ and $\delta K$, is surrounded by a vacuum region which contributes to the vaccum energy $\delta W_{v}$. The resistive wall (vacuum vessel) contributes to $D_{w}$. The feedback coils that contribute to the coil excitation energy $\delta E_{c}$ are located both inside (the I-coils) and outside (the C-Coils) of the vacuum vessel.

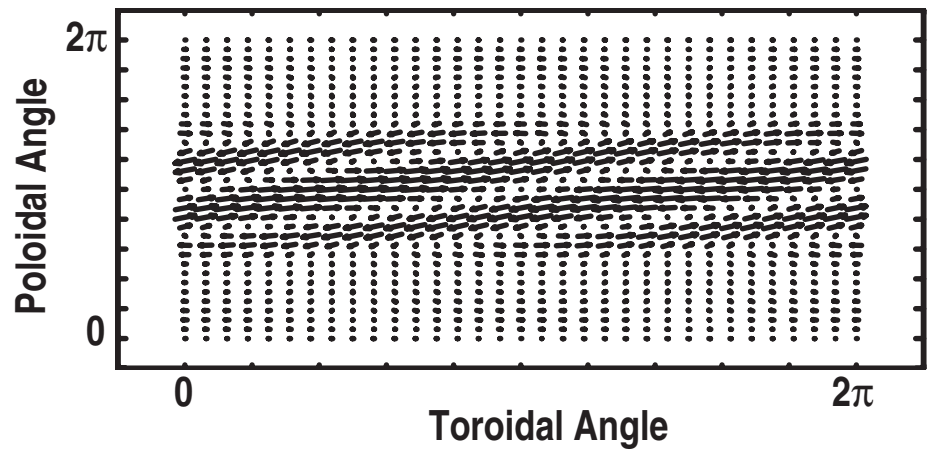

Fig. 2. Distribution of the eddy current of the unstable RWM on the surface of the resistive wall unfolded into the toroidal-poloidal plane. It is seen that the perturbed current is concentrated near the poloidal angle $\pi$, i.e. the outboard midplane. 
In Eq. (3), $\tau_{c c^{\prime}}$ is determined by the self and mutual inductances of the coils, $F_{l i}$ is the sensor matrix which measures the magnetic fluxes induced by the eigenmodes in the sensor loop $l$, and $G_{c l}$ is the gain matrix. The stability of the feedback is completely described by Eqs. (2) and (3) above and the closed loop feedback problem is reduced to a small set of coupled lumped circuit equations. This set of equations is, in general, non-self-adjoint. For feedback with a single array of sensors and a single array of feedback coils, the stability may be studied by using the method of Nyquist diagram ${ }^{13}$ and for multiple sensor arrays and multiple feedback coils, the characterisitc of Eqs. (2) and (3) have to be solved.

Shown in Fig. 3 is the Nyquist diagrams of transfer functions $P(s)$ for equilibria with different $C_{\beta}$ 's. Here s is the varialbe for Laplace transform, and $P(s)$ is given by

$$
P(s)=\sum_{i} \frac{F_{i} E_{i}}{s-\gamma_{i}}
$$

and $C_{\beta}$ is defined by

$$
C_{\beta}=\frac{\beta_{N}-\beta_{N}^{N W}}{\beta_{N}^{I W}-\beta_{N}^{N W}}
$$

$C_{\beta}$ is a measure of the extent to which the $\beta$ value is above the no wall limit. $C_{\beta}=0$ for plasma at the no wall $\beta$ limit and $C_{\beta}=1$ for plasma at the ideal wall $\beta$ limit. The curves are symmetric to the horizontal axis. Only the upper half of the curves are shown. Stable equilibria have their curves encircle $(-1 ., 0$.$) . On the left is for feedback with radial field$ sensors and on the right is for feedback with poloidal field sensors. It is seen that feedback with poloidal field sensors is much more effective than with radial field sensors. One of the advantages of using the normal mode approach is that the set of open loop eigenfunctions are independent of the feedback configuration. We may easily rearrange the sensor locations, the feedback scheme or the feedback coil arrangements without having to revisit the open loop stability problem. For instance, the internal feedback coils have recently been installed on DIII-D. ${ }^{16}$ The difference in performance of the internal I-Coils versus the external C-Coils can be evaluated for the same set of reference equilibria. Shown in Fig. 4 is the ratio of the excitation matrix elements from the RWM for this set of equilibria due to the I-coils and C-coils as a function of $C_{\beta}$. This represents the ratio of the effectiveness to input or extract energy from the I- versus C-coils from the unstable RWM. We see that on the average the I-Coils are a factor of 4 to 5 times more effective than the C-Coils. 


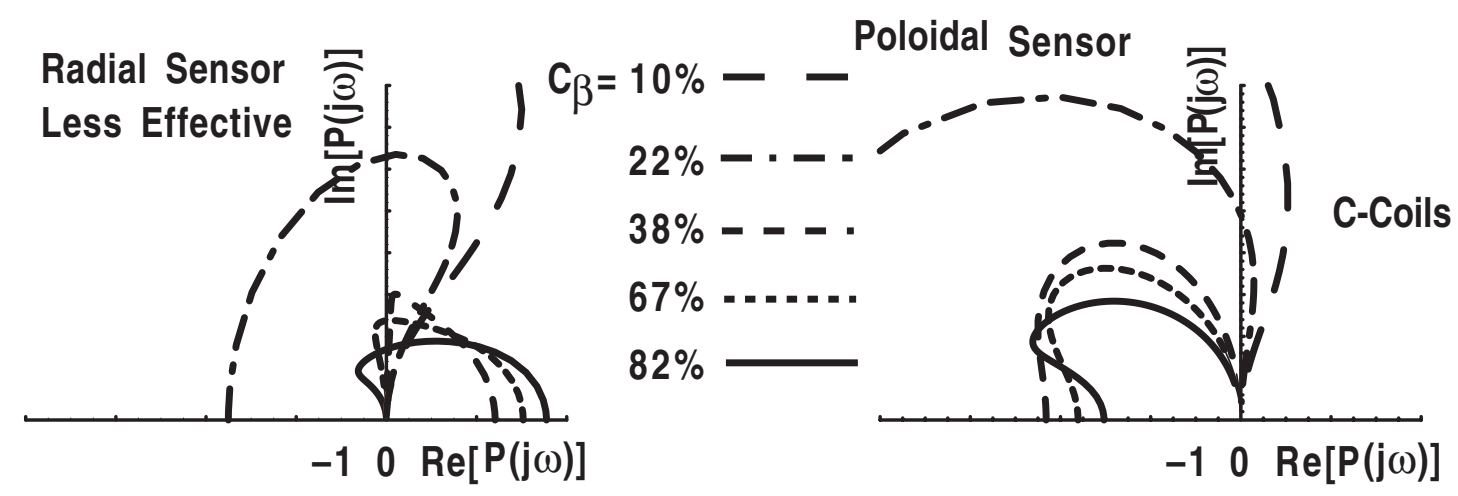

Fig. 3. Nyquist diagrams of transfer functions for equilibria with different $C_{\beta}$. The curves are symmetric to the horizontal axis. Only the upper half of the curves are shown. Stable equilibria have their curves encircle $(-1,0$.$) . On the left is for feedback with radial field sensors and on the right is for feedback with poloidal$ field sensors. It is seen that feedback with poloidal field sensors is much more effective than with radial field sensors.

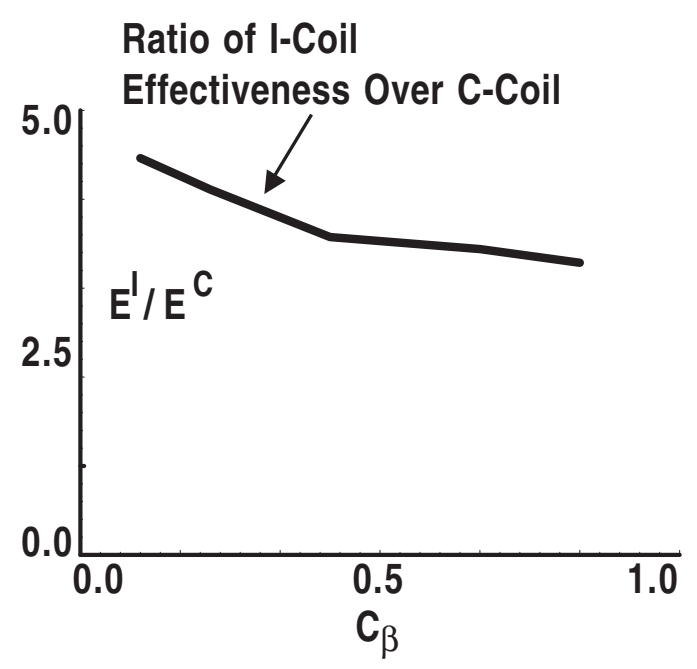

Fig. 4. The ratio of the excitation matrix elements from the RWM for the reference set of equilibria due to the I-coils and C-coils as a function of $C_{\beta}$. This represents the ratio of the effectiveness to input or extract energy from the I- versus C-coils from the unstable RWM. 


\section{STABILIZATION OF THE RWM BY PLASMA ROTATION AND DISSIPATION}

It was first predicted by Bondeson and Ward ${ }^{11}$ that plasma rotation, coupled with the inherent dissipation within the plasma, can provide stabilization to the RWM with a plasma rotation at a few percent of the Alfven frequency. This has been observed and provided a stable operation path to reach the ideal wall $\beta$ limit for DIII-D. ${ }^{4}$ This stabilization process can be modeled by using MARS, a linear non-ideal MHD code which includes a non-uniform plasma rotation $\Omega$.

MARS solves the following set of equations

$$
\begin{gathered}
\rho(\gamma+i n \Omega) \vec{v}_{1}=-\vec{\nabla} p_{1}+\vec{j}_{1} \times \vec{B}_{0}+\vec{j}_{0} \times \vec{b}_{1}-\vec{\nabla} \cdot \vec{\Pi}_{1}-\rho \vec{U}\left(\vec{v}_{1}\right) \\
(\gamma+i n \Omega) \vec{b}_{1}=\vec{\nabla} \times\left(\vec{v}_{1} \times \vec{B}_{0}-\eta \vec{j}_{1}\right)+\left(\vec{b}_{1} \cdot \vec{\nabla} \Omega\right) R^{2} \vec{\nabla} \phi \\
\vec{j}_{1}=\vec{\nabla} \times \vec{b}_{1} \\
(\gamma+i n \Omega) p_{1}=-\left(\vec{v}_{1} \cdot \vec{\nabla}\right) p_{0}-\Gamma p_{0} \vec{\nabla} \cdot \vec{v}_{1} \\
(\gamma+i n \Omega) \rho_{1}=-\left(\vec{v}_{1} \cdot \vec{\nabla}\right) \rho_{0}-\rho_{0} \vec{\nabla} \cdot \vec{v}_{1}
\end{gathered}
$$

In the above set of equations, $\frac{\partial}{\partial t}=\gamma$ is the complex growth rate, and the toroidal variation of the perturbed quantities is related by $\frac{\partial}{\partial \phi}=i n$ to the toroidal mode number $\mathrm{n}$. The density equation Eq. (10) is decoupled from the rest. Therefore can be solved separately. The viscous stress tensor $\vec{\Pi}_{1}$ results from fluid approximations to the ion Landau damping.

A general model dispersion relation may be derived ${ }^{17}$ for the above set of equations,

$$
(\gamma+i n \Omega)^{2} K+(\gamma+i n \Omega) D+\delta W_{p}+\frac{\delta W_{v}^{b} \gamma \tau_{w}+\delta W_{v}^{\infty}}{\gamma \tau_{w}+1}=0
$$

This equation is cubic in the growth rate $\gamma$ and in general possesses three solution. The first one of these has its origin coming from the unstable MHD mode, the external kink; the second one has its origin from the flux diffusion through the resistive wall - it is a stable mode in the absence of plasma or the external coils; the third one is the stable companion to the first mode. In the limit of an ideally conducting external wall, the first mode is stabilzed by the external wall. There is no instability. The second mode has its damping rate reduced to zero. When the resisitivity of the conducting wall is taken into account, the unstable kink couples with the flux diffusion to change the character of the external kink and the flux diffusion to the unstable RWM and a different stable flux diffusion mode. With the inclusion of plamsa dissiptaion $D$ and the plasma rotation the coupling of these two modes are modified again by the second term. This coupling leads to the stabilization of the RWM. 
Throughout this process, the third mode is never quite heavily involved. The third mode is eliminated by neglecting the kinetic energy term to obtain the following dispersion relation.

$$
(\gamma+i n \Omega) \tilde{D}+\delta W_{p}+\frac{\delta W_{v}^{b} \gamma \tau_{w}+\delta W_{v}^{\infty}}{\gamma \tau_{w}+1}=0
$$

Topology of the stability phase space in the $\Omega-C_{\beta}$ plane of the model dispersion relation Eq. (11) is shown in Fig. 5. The weak dissipation limit, i.e., when $D$ is small is shown on the left. It is characterized by the negative slope of the stability boundary, i.e., plasma with the smallest $C_{\beta}$ requires the largest rotation velocity for its stabilization. The strong dissipation limit is shown in the center. It is characterized by that the maximum value of $\Omega$ for stabilization occurs at a mid-range of $C_{\beta}$ value. The diagram is very similar to that on the right, in this case the second order dispersion relation Eq. (12), i.e. neglecting the kinetic energy, is solved. We observe that the strong dissipation limit is very similar to neglecting the kinetic energy term. The structure of these topological shapes are also obtained independently by Fitzpatrick. ${ }^{18}$

\section{A. Damping Models for RMW}

To complete the prescription of model for the MARS code, an explicit description for the viscosity tensor needs to be specified. In the following, two distinct models are described. The first one is the ion sound wave damping model first developed by Hammet and Perkins. ${ }^{19}$

$$
\begin{gathered}
\vec{\nabla} \cdot \vec{\Pi}=\kappa_{\|} \sqrt{\pi}\left|k_{\| \mid} v_{t h_{i}}\right| \rho \vec{v}_{1} \cdot \hat{b} \hat{b} \\
\kappa_{\|}=0.5
\end{gathered}
$$

The second one is the kinetic damping model. This model was used before to study the damping of the TAE modes by Bondeson and Chu. ${ }^{20}$ The kinetic damping model utilizes the kinetic energy principle with $\omega *=0, \omega_{D}=0$

$$
\begin{gathered}
\Delta W_{M H D}=\Delta W_{p}(\vec{\xi}, \Gamma=0)+\Delta W_{k}(\vec{\xi}) \\
\Delta W_{k}=\sum_{j}\left(\Delta W_{T_{j}}+\Delta W_{c_{j}}\right) \\
\Delta W_{c}=\int_{\text {circ. }} d \vec{x} d \vec{v}\left(-\frac{\partial f}{\partial E}\right) \frac{\omega}{\omega-\left(n q-m^{\prime}\right) \omega_{t}}\left|<\exp \left(i \chi_{m}^{\prime}\right) H>\right|^{2} \\
\Delta W_{t}=\int_{\text {trapped }} d \vec{x} d \vec{v}\left(-\frac{\partial f}{\partial E}\right) \frac{\omega}{\omega+m^{\prime} \omega_{b}}\left|<\exp \left(i \chi_{m}^{\prime}\right) H>\right|^{2}
\end{gathered}
$$



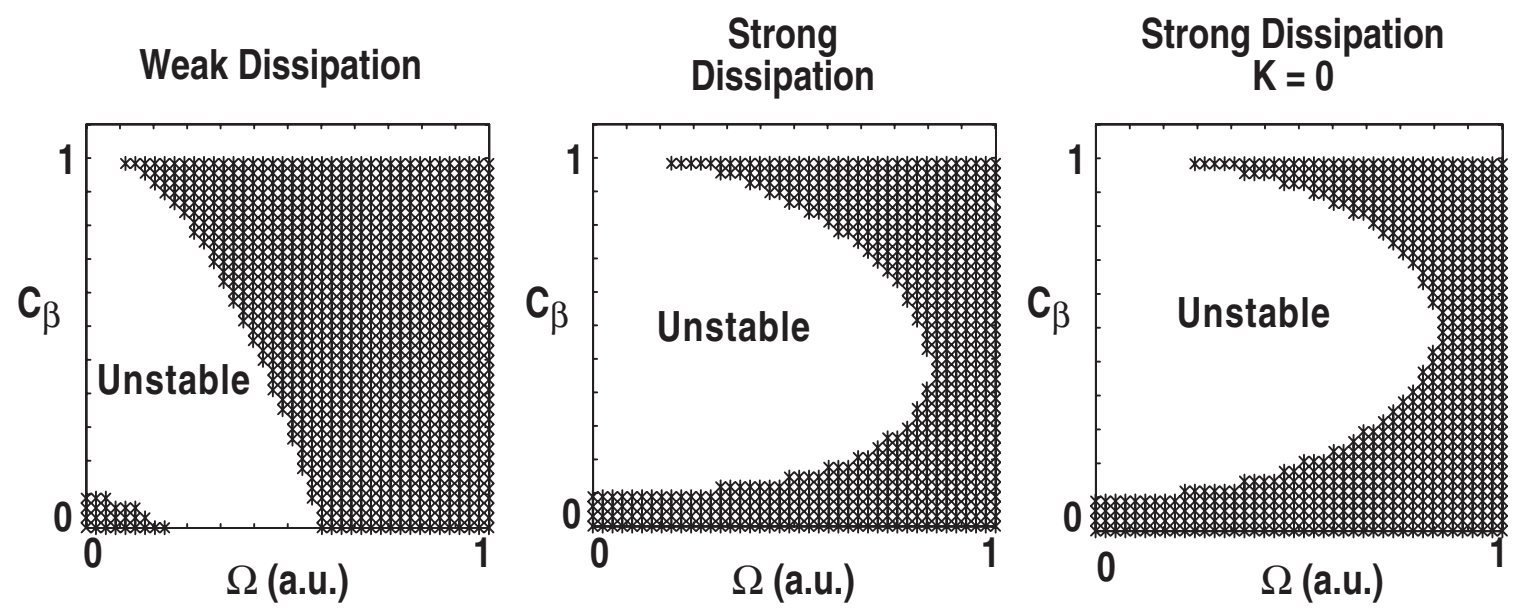

Fig. 5. Topology of the unstable phase space in the $\Omega-C_{\beta}$ plane of the model dispersion relation. The weak dissipation limit is shown on the left. It is characterized by the negative slope of the stability boundary, i.e., plasma with the smallest $C_{\beta}$ requires the largest rotation velocity for stabilization. The strong dissipation limit is shown in the center. It is characterized by the maximum value of $\Omega$ for stabilization occurs at the mid-range of $C_{\beta}$ value. The center diagram is very similar to that on the right, in which the kinetic energy term is neglected.

$$
H=\mu Q_{L}+m u_{\|}^{2 \vec{\xi} \cdot \kappa}
$$

In Eq. (15) $\left(\Delta W_{M H D}, \Delta W_{p}, \Delta W_{k}\right)$ is the (total, fluid contribution to, kinetic contribution to) perturbed potential energy, $\Gamma$ is the adiabatic index of the plasma. In Eq. (16), the kinetic contribution to the potential energy $\Delta W_{k}$ is further decomposed into the contributions from each species $j$ due to the trapped particle $\Delta W_{T_{j}}$; and the circulating particles $\Delta W_{c_{j}}$. Landau damping results from the resonant denominators in Eqs. (17) and (18). $H$ is the perturbed energy of the particle, $\mu$ in its magnetic moment, $Q_{L}$ is the perturbed magnetic field in the Lagragian frame moving with the perturbed magnetic field line, $\vec{\kappa}$ is the field line curvature, $\left(\omega_{b}, \omega_{t}\right)$ are the (trapped particle bounce frequency, circulating particle transit frequency). Note that although Eq. (15) is written in terms of the perturbed potential energy of the plasma, it gives an equivalent radial force with the imaginary part giving rise to the damping of the RWM. The more exact detailed explanation of this equivalent relationship between the potential energy description and the fluid description is given in Eqs. (10-14) of Ref. 20. The kinetic damping model was first used in the work of Ref. 21.

The MARS code has been used to study the effect of plasma rotation on the stability of the RWM for the reference set of equilibria. Results utilizing either the kinetic damping model and the ion sound wave damping model has been obtained. For simplicity, a uniform 
plasma rotation profile is employed. The results are compared with the experimental results $^{22}$ from DIII-D. Shown in Fig. 6 is the comaparison of the stability boundary computed using MARS with two different plasma dissipation models with the critical rotational speed from the DIII-D RWM experiments. It is seen that the MARS curves from both damping models show the characteristics of strong damping limit. This is more obvious for the ion sound wave damping model. But we note that the kinetic damping model gives even stronger damping. It is also observed that the results from MARS with the ion sound wave damping model agrees with the experimental results qualitatively; whereas the computed required rotation from the kinetic damping model is too low. However, we deem the present comparison not conclusive in determining the proper damping model appropriate for the experiment. More careful analysis is required. Several of the obvious sources of discrepancies are: the deviation of the equilibria in the experiment from the reference set is non-negligible; the rotation profiles are quite different; or the appropriate damping model is not used. In the present attempt to compare the stability diagrams inferred from both the experimental data and that from the modeling, due to the large range of discharge conditions involved, only the most prominent dependencies on the stability diagrams have been taken into account. The $C_{\beta}$ values of experimental data are inferred from their equilibrium values of $\beta$ and self-inductances $\ell_{i}$, and not from the more tedious computation of ideal stability inferred from individual reconstructed equilibria. While it is expected that this will invariably introduce extra scatter into the stability diagram, even to cause plasma equilibria to range above the ideal wall $\beta$ limit, or require the plasma to possess a finite rotation below the no wall $\beta$ limit for RWM stabilization, we still deem this comparison the first necessary step in understanding the landscape of the rotational stabilization of the RWM. The approach is complementary to that of focussing attention on only a few shots with a detailed comparison. This is due to the inherent uncertainties in the experimental measurements and equilibrium reconstruction. Results from present comparison are therefore not conclusive. We note that it is possible that the detected RWM is in a nonlinear state in which the flux surface deformation could be non-negligible. Then a large additional damping of the plasma angular momentum could be described by the work of Shaing. ${ }^{23}$

The behavior of the normalized growth rate as a function of $C_{\beta}$ with different plasma rotation, for different wall locations is shown in Fig. 7. Ion sound wave damping model and uniform plasma rotation is utilized. Shown in the center are results computed using the DIII-D vacuum vessel. It is seen that increasing plasma rotation, the RWM is gradually stabilized. The stabilization occurs first at the lowest and highest range of $C_{\beta}$ values. When $\Omega / \omega_{A} \geq 2 \%$, the RWM is stabilized over the whole range of $C_{\beta}$. Results for vacuum vessel one third farther away is shown on the right. It is seen that with the wall farther away, the growth rates of RWM become larger with no plasma rotation, but require the least amount of rotation to stabilize. Results with the wall one third closer is shown on the left. With a closer wall, the value of $\gamma \tau_{w}$ becomes smaller, yet the higher range of $C_{\beta}$ values becomes more difficult to stabilize. 


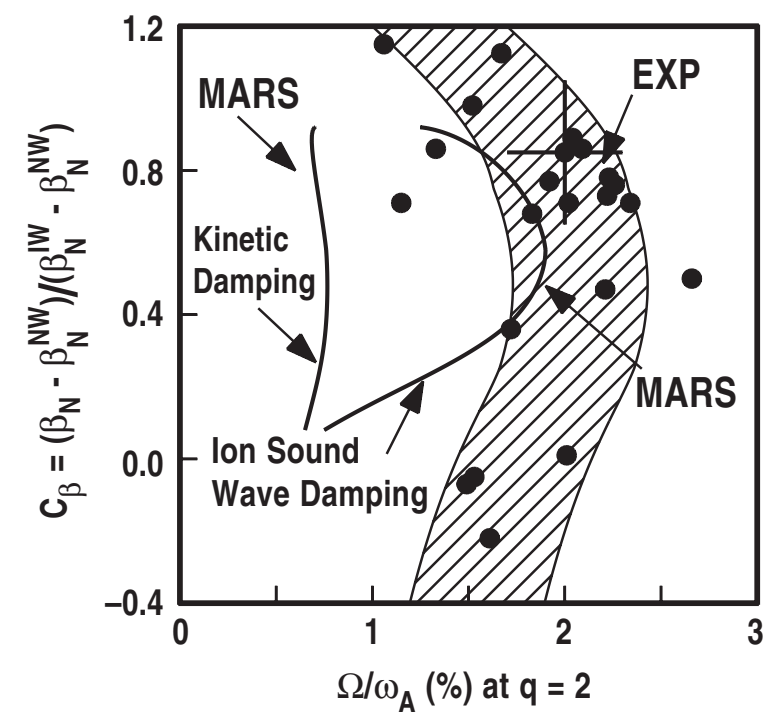

Fig. 6. A broad ranged comparison of the stability boundary computed for a model set of equilibria using MARS with two different plasma dissipation models with the experimental results from DIII-D. The stability properties of the experimental equilibria are inferred from their equilibrium properties rather than from detailed stability calculation. This is expected to introduce scatter in the comparison. It is seen that the results from MARS with the ion sound wave damping model agrees with the experimental results qualitatively; whereas the requirement given by the kinetic damping model is too low.
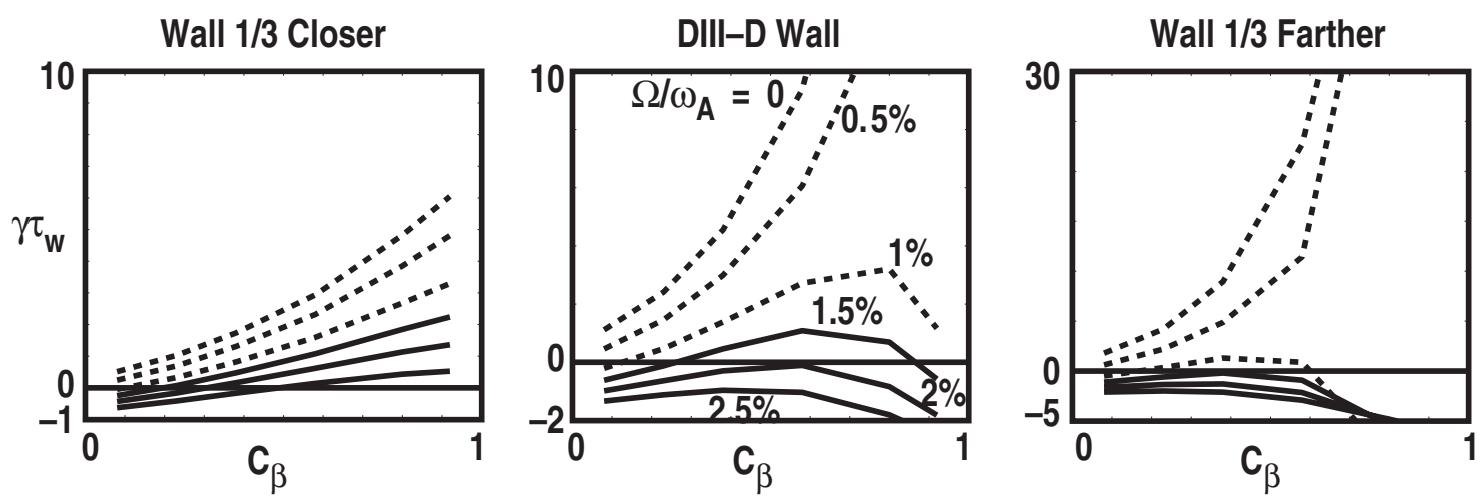

Fig. 7. Shown in the center is the normalized growth rate as a function of $C_{\beta}$ for different plasma rotation. It is seen that increasing plasma rotation gradually stabilizes the RWM. It is interesting to note that the stabilization occurs first at the lowest and highest range of $C_{\beta}$ values. When $\Omega / \omega_{\mathrm{A}} \geq 2 \%$, the RWM is stabilized over the whole range of $C_{\beta}$. With the wall farther away (the right panel), the growth rate of RWM becomes larger with no flow, but requires less rotation to stabilize. However, with a closer wall (the left panel), the value of $\gamma \tau_{w}$ becomes smaller, yet the higher range of $C_{\beta}$ values becomes more difficult to stabilize. 


\section{FEEDBACK STABILIZATION OF THE RWM IN A ROTATING PLASMA}

To compare the MARS-F code and the NMA code in their ability to treat feedback, the MARS-F code was first employed to study the feedback stabilization of the reference set of equilbria in the limit of ideal plasma with no rotation.

Shown in Fig. 8 on the left is the Nyquist diagram for the poloidal and radial sensors for the equilibrium with $C_{\beta}=58 \%$ and its comparsion with the NMA code. It is seen that using poloidal sensors stabilizes the RWM whereas using radial sensors does not. We notice that the two approaches do not give exactly the same transfer functions. This could result from the use of different approaches or the details of the implementation of the sensor and feedback geometry. Shown on the right is the variation of $\gamma \tau_{w}$ as a function of feedback gain for equilibria with different $C_{\beta}$ values and using poloidal sensors. It is seen that the RWM is stabilized over the whole range of $C_{\beta}$ values, in agreement with the results from the NMA code. We note that in a separate work, ${ }^{24}$ it has also been verified that MARS observes the same ideal stability boundary as DCON and the VACUUM code.

In summary, MARS-F and NMA agree on values of $\beta_{N}^{N W}$ and $\beta_{N}^{I W}$. The two methods agree on the effect of feedback over the range $0 . \leq C_{\beta} \leq 92 \%$.

A natural consequence of the presence of the dissipation effect on the plasma with rotation under the feedback action is its effect on the response of the plasma to an external perturbation. ${ }^{25,26}$ The response to a static perturbation and the response to a dynamic perturbation are related. ${ }^{27}$ These considerations predicted the existence of very low frequency plasma resonances and are observed in the plasma very low frequency experiments of Reimerdes et al. ${ }^{28}$

Next the effect of flow on feedback for this set of equilibria is analyzed. The results are shown in the following two figures. In Fig. 9, we see that the normalized growth rates are reduced by the synergistic effect of plasma rotation and feedback. Shown on the left is the growth rate as a function of the feedback gain $G$ for the equilibrium with $C_{\beta}=38 \%$ using different values of $\Omega / \omega_{A}$. Shown on the right is the variation of $\gamma \tau_{w}$ with respect to the feedback gain using radial sensors. It is seen that increasing gain is the most effective below $G=1$ to 2 , above which it becomes less effective. (Here feedback gain $G$ is defined as the ratio of the perturbation field detected at the sensor due to the feedback coil vs. the total field detected at the sensor.)

Results in Fig. 10 show plasma rotation reduces the values of feedback gain required for the stabilization of the RWM. The feedback gain is plotted as a function of $\Omega / \omega_{A}$ for different $C_{\beta}$ values. It is seen that for both poloidal and radial sensors, the reduction in gain is monotonic with the rotation speed. For radial sensors, feedback stabilization becomes effective only after rotation has reduced the growth rate sufficiently. 

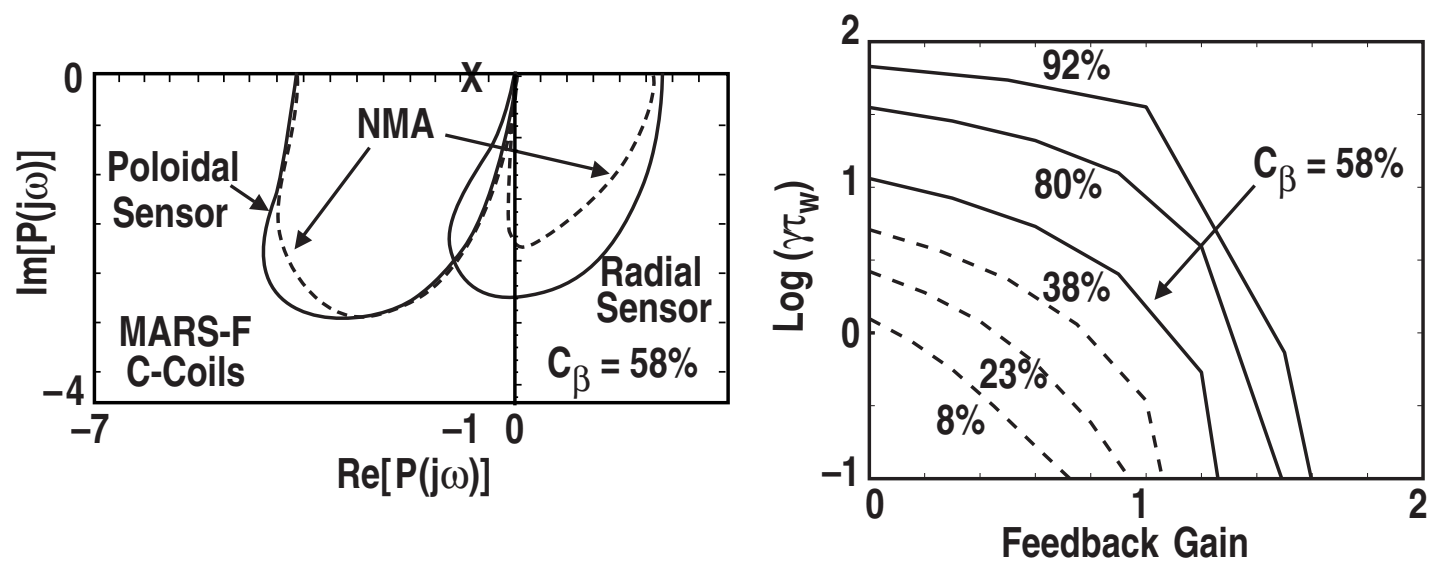

Fig. 8. Shown on the left is the Nyquist diagram for the poloidal and radial sensors for an equilibrium with $C_{\beta}=58 \%$ and the comparison with the NMA code. It is seen that using poloidal sensors stabilizes the RWM whereas using radial sensors does not. Shown on the right is the variation of $\gamma \tau_{w}$ as a function of feedback gain for equilibria with different $C_{\beta}$ values and using poloidal sensors. It is seen that the RWM is stabilized over the whole range of $C_{\beta}$ values, in agreement with the results from the NMA code.

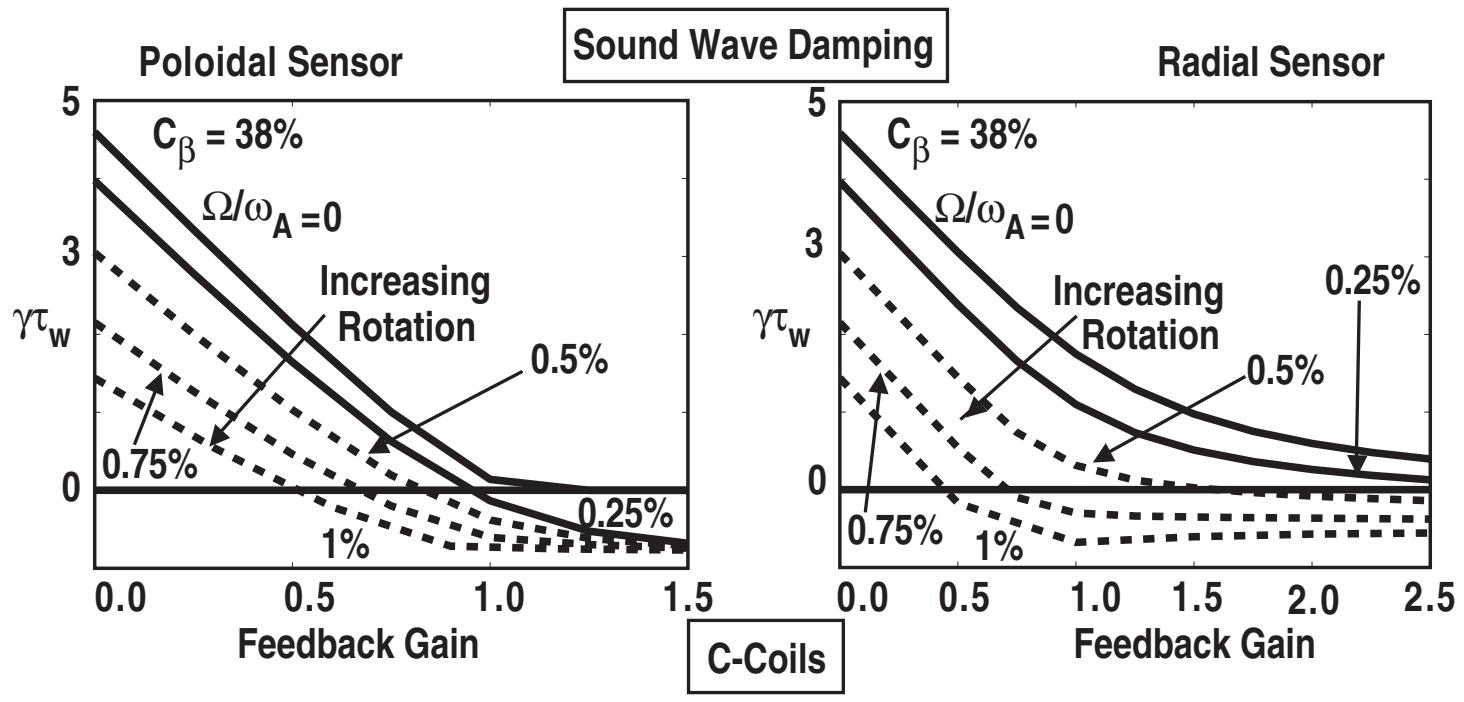

Fig. 9. The normalized growth rates are reduced by the synergistic effect of plasma rotation and feedback. Shown on the left is the growth rate as a function of the feedback gain for the equilibrium with $C_{\beta}=38 \%$ using different values of $\Omega / \omega_{\mathrm{A}}$. Shown on the right is the variation of $\gamma \tau_{w}$ with respect to the feedback gain using radial sensors. It is seen that increasing gain is the most effective below $G=1$ to 2 , above which it becomes less effective. 


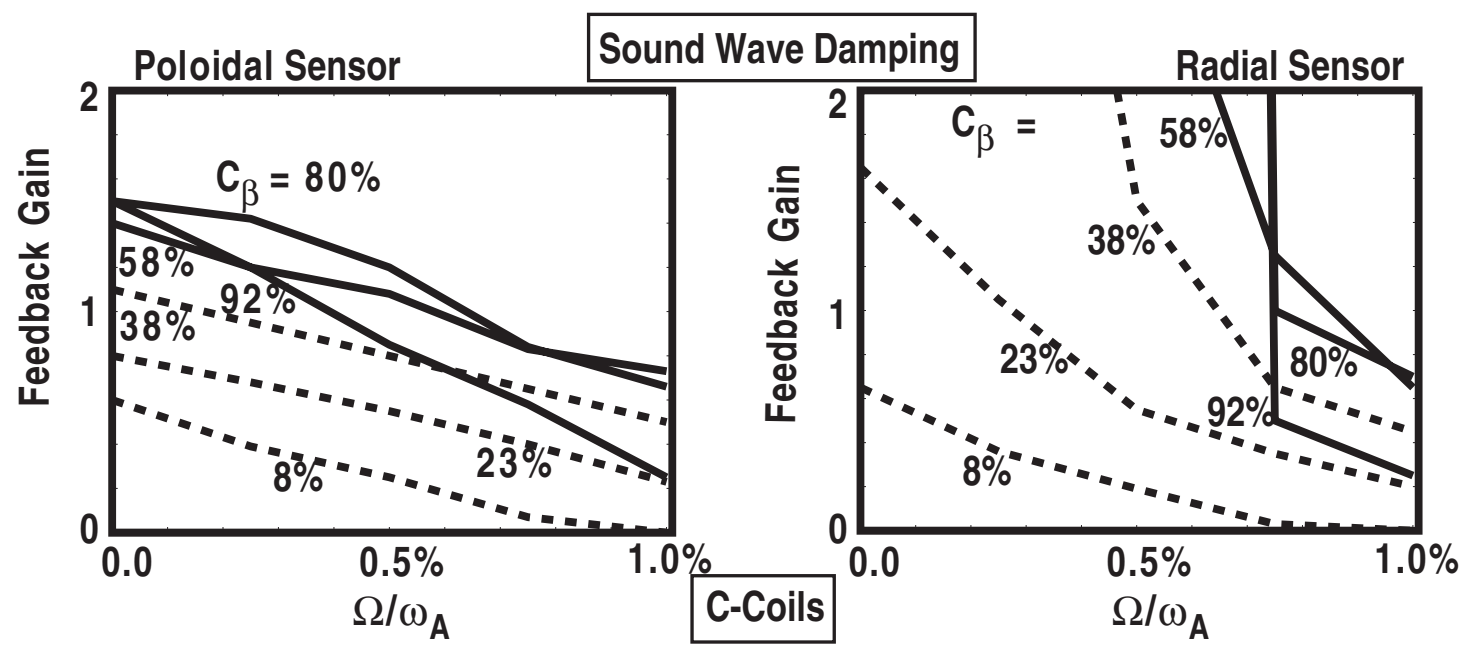

Fig. 10. Diagram showing plasma rotation reduces the values of feedback gain required for the stabilization of the RWM. The feedback gain is plotted as a function of $\Omega / \omega_{\mathrm{A}}$ for different $C_{\beta}$ values. It is seen that for both poloidal and radial sensors, the reduction in gain is monotonic with the rotation speed. For radial sensors, feedback stabilization becomes effective only after rotation has reduced the growth rate sufficiently. 


\section{FEEDBACK AND ROTATION STABILIZATION OF THE RWM IN ITER-SCENARIO IV}

The ITER scenario IV is an advanced tokamak with the following basic design parameters: $I_{p}=9 \mathrm{MA}, R=6.35 \mathrm{~m}, a=1.85 \mathrm{~m}, \kappa_{\text {sep }}=1.97, \delta_{\text {sep }}=0.58, \beta_{N_{\text {design }}}=2.57$, $\beta_{N}^{N W}=2.45$, and $\beta_{N}^{I W}=3.65$. In this scenario, without feedback or plasma rotation, the resistive wall mode is unstable.

The feedback geometry is shown in Fig. 11 in which the designed plasma surface and the designed non-uniform double wall, as well as the positions of the active and sensor coils are shown. Dashed line indicates the plasma facing component (the blanket), modeled as a complete thin wall. These are all included in the study. ${ }^{15}$

The effect of feedback on the stabilization of the RWM is shown in Fig. 12. Plotted are the Nyquist diagrams of the transfer function for different plasma $C_{\beta}$ values. Poloidal sensors are used. It is seen that a $C_{\beta}$ value up to $50 \%$ can be stabilized. More detailed study indicates that even higher $C_{\beta}$ values may be stabilized by the judicious design of an optimal controller. Shown in Fig. 13 is the Nyquist plot of the transfer function for three different rotation speeds, with uniform rotation profile and the ion sound wave damping model units $\kappa_{\|}=0.5$. It is seen that plasma rotation further stabilizes the RWM. 


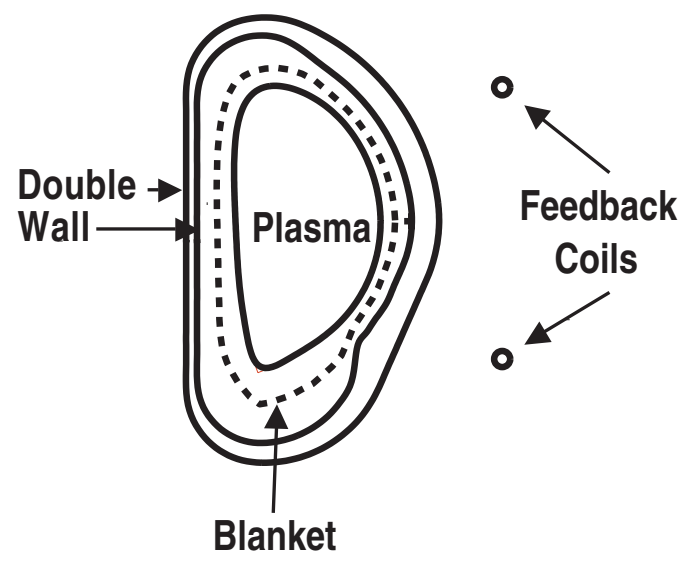

Fig. 11. The configuration used for the feedback study of the RWM in ITER. Shown are the plasma surface, the designed non-uniform double wall, as well as the positions of the active and sensor coils. Dashed line indicates the plasma facing component (the blanket), modeled as a complete thin wall.

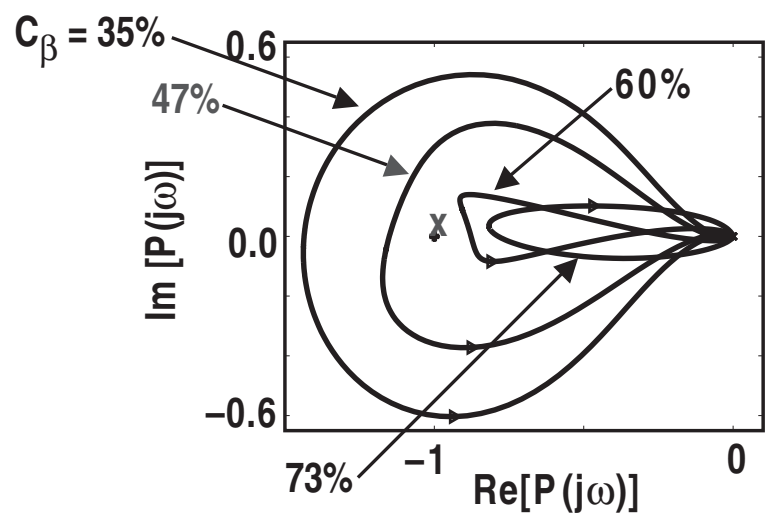

Fig. 12. Nyquist diagrams of the transfer function for different plasma pressure. Poloidal sensors are used.

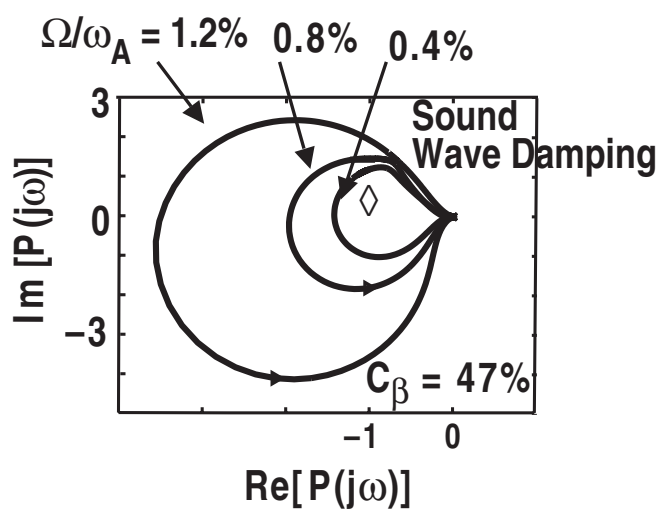

Fig. 13. Nyquist plot of the transfer function for three different rotation speeds, with uniform rotation profile and the ion sound wave damping model. 


\section{CONCLUSION}

RWM is expected to be present in future reactor designs based on the advanced tokamak concept. At the present moment, both rotation and feedback stabilization have been demonstrated to be effective in stabilizing the resistive wall mode. Although future reactors are not expected to be rotating at a high rotation frequency, to properly evaluate the effect of electromagnetic feedback and the effect of rotation on the future reactors, a comprehensive model which encompasses both feedback and rotation is needed. The complete response of an ideal plasma has been utilized to study the feedback stabilization of RWM in general geometry. Poloidal sensors have been found to be superior than radial sensor and provides stabilization of plasma up to $90 \%$ of the ideal wall $\beta$ limit. This provides a pessimistic limit to plasma with flow. Rotation, together with dissipation, has been predicted to be effective in stabilizing the resistive wall mode. The effect of rotational stabilization of the plasma depends on the dissipation model assumed. Broad qualitative features of the experiment have been reproduced. Detailed quantitative tests of the validity of the damping models remain. The stability phase space has been found to be in qualitative agreement with the marginial stability value measured in the experiment. The MARS-F code has been ultilized to study the effect of feedback with a rotating plasma. First, the MARS-F code has been benchmarked in the ideal response limit without rotation against the ideal NMA code. MARS-F is then utilized to study the requirement of feedback in a rotating plasma with dissipation. Rotation reduces the feedback gain required for stabilization. The reduction is significant when the plasma rotation appraoch the critical rotation speed. Finally, the proposed design in ITER can be feedback stabilized up to $C_{\beta}=50 \%$ above the no wall $\beta$ limit. 


\section{REFERENCES}

${ }^{1}$ C. Kessel, J. Manickam, G. Rewolt, and W.M. Tang, Phys. Rev. Lett. 72, 1212 (1994).

${ }^{2}$ A.D. Turnbull, Y.R. Lin-Liu, H.E. St. John, T.S. Taylor, Phys. Rev. Lett. 74, 718 (1995).

${ }^{3}$ E.J. Strait, J. Bialek, I.N. Bogatu, et al., "Resistive wall mode stabilization with internal feedback coils in DIIID," this conference (2003).

${ }^{4}$ A.M. Garofalo, E.J. Strait, L.C. Johnson, R.J. LaHaye, E.A. Lazarus, G.A. Navratil, M. Okabayashi, J.T. Scoville, T.S. Taylor, and A.D. Turnbull, Phys. Rev. Lett. 89, 235001 (2002).

${ }^{5}$ M. Okabayashi, J. Bialek, M.S. Chance, et al., Plasma Phys. and Control. Fusion 44, B339 (2002).

${ }^{6}$ R. Aymar, P. Barabaschi, Y. Shimomura for the ITER Team, Plasma Phys. Control. Fusion 44, 519 (2002).

${ }^{7}$ M.S. Chu, M.S. Chance, A. Glasser, and M. Okabayashi, Nucl. Fusion 43, 441 (2003).

${ }^{8}$ A.H. Glasser and M.S. Chance, Bull. Am. Phys. Soc. 42, 1848 (1997).

${ }^{9}$ M.S. Chance, Phys. Plasmas 4, 2161 (1997).

10 J.L. Luxon, Nucl. Fusion 42, 614 (2002).

11 A. Bondeson and D.J. Ward, Phys. Rev Lett. 72, 2709 (1994).

12 A. Bondeson, G. Vlad, H. Lutjens, Phys. Fluids B 4, 1889 (1992).

${ }^{13}$ Y.Q. Liu, A. Bondeson, C.M. Fransson, B. Lennartson, and C. Breitholtz, Phys. Plasmas 7, 3681 (2000).

${ }^{14}$ Y.Q. Liu, A. Bondeson, Phys. Rev. Lett. 84, 907 (2000).

${ }^{15}$ Y.Q. Liu, A. Bondeson, Y. Gribov, and A. Polevoi, "Stabilization of the Resistive Wall Mode in ITER by Active Feedback and Toroidal Rotation," to be printed in Nucl. Fusion (2003).

${ }^{16}$ G.L. Jackson, P.M. Anderson, J. Bialek, et al., "Initial Results from the New Internal Magnetic Field Coils for Resistive Wall Mode Stabilization in the DIII-D Tokamak," in the Proceedings of the 30th European Conference on Controlled Fusion and Plasma Physics, St. Petersburg, Russia, 2003 (European Physical Society) paper P-4.47 to be published on CD-ROM.

${ }^{17}$ M.S. Chu, J.M. Greene, T.H. Jensen, R.L. Miller, A. Bondeson, R.W. Johnson, and M.E. Mauel Phys. Plasmas 2, 2236 (1995).

${ }^{18}$ R. Fitzpatrick, Phys. Plasmas 9, 3459 (2002). 
${ }^{19}$ Hammet and Perkins, Phys. Rev. Lett. 64, 3019 (1990).

${ }^{20}$ A. Bondeson and M. S. Chu, Phys. Plasmas 3 , 3013 (1996).

${ }^{21}$ A. Bondeson, Y.Q. Liu, D. Gregoratto, C.M. Fransson, and Y. Gribov, Plasma Phys. and Control. Fusion 45, A253 (2003).

${ }^{22}$ R.J. LaHaye, M.S. Chu, E.J. Strait, A.M. Garofalo, H. Reimerdes, M. Okabayashi, Bull. Am. Phys. Soc. 47, 274 (2002).

${ }^{23}$ K. C. Shaing, Phys. Plasmas 10, 1443 (2003).

${ }^{24}$ J. Meynard, Bull. Am. Phys. Soc. 48, 18 (2003).

${ }^{25}$ A.H. Boozer, Phys. Rev. Lett. 86, 1176 (2001).

${ }^{26}$ A.M. Garofalo, T.H. Jensen, E.J. Strait, Phys. Plasmas 9, 4573 (2002).

${ }^{27}$ M.S. Chu, V.S. Chan, M.S. Chance, et al., Nucl. Fusion 43, 196 (2003).

${ }^{28}$ H. Reimerdes, M.S. Chu, A.M. Garofalo, G.L. Jackson, R.J. La Haye, G.A. Navratil, M. Okabayashi, J.T. Scoville, E.J. Strait, submitted to Phys. Rev. Lett. (2003). 


\section{ACKNOWLEDGMENTS}

Work supported by the U.S. Department of Energy under DE-FG03-95ER54309, DEAC0276CH03073, and DE-FG02-89ER54461. 